\title{
Some Bounds for the Kirchhoff Index of Graphs
}

\author{
Yujun Yang \\ School of Mathematics and Information Science, Yantai University, Yantai 264005, China \\ Correspondence should be addressed to Yujun Yang; yangyj@yahoo.com
}

Received 7 February 2014; Accepted 24 June 2014; Published 10 July 2014

Academic Editor: Massimo Furi

Copyright (c) 2014 Yujun Yang. This is an open access article distributed under the Creative Commons Attribution License, which permits unrestricted use, distribution, and reproduction in any medium, provided the original work is properly cited.

\begin{abstract}
The resistance distance between two vertices of a connected graph $G$ is defined as the effective resistance between them in the corresponding electrical network constructed from $G$ by replacing each edge of $G$ with a unit resistor. The Kirchhoff index of $G$ is the sum of resistance distances between all pairs of vertices. In this paper, general bounds for the Kirchhoff index are given via the independence number and the clique number, respectively. Moreover, lower and upper bounds for the Kirchhoff index of planar graphs and fullerene graphs are investigated.
\end{abstract}

\section{Introduction}

Let $G$ be a connected graph with vertices labeled as $1,2, \ldots, n$. It is natural to view $G$ as an electrical network by imagining each edge of $G$ to be a (unit) resistor. In this guise, it is reasonable to consider the effective resistance between any two vertices of $G$, and the novel concept of resistance distance $[1] r_{i j}(G)$ between any two vertices $i$ and $j$ of $G$ is thus defined as the effective resistance between them. Compared to the (shortest-path) distance $d_{i j}(G)$ between $i$ and $j$ in $G$, it is well known that $r_{i j}(G) \leq d_{i j}(G)$ with equality if and only if $i$ and $j$ are connected by unique path [1].

There are many distance-based molecular structure descriptors, as reviewed in [2], which have played important roles in QSAR and QSPR. Among these structure descriptors, the most famous one is the Wiener index $W(G)$ [3], which is known as the sum of distances between all pairs of vertices. Analogous to the Wiener index, the Kirchhoff index of $G$ $[1,4]$, denoted by $K f(G)$, is defined as the sum of resistance distances between all pairs of vertices; that is,

$$
K f(G)=\sum_{i<j} r_{i j}(G) .
$$

As summarized in [2], much work has been done by many researchers to investigate bounds for the Kirchhoff index. There are not only general bounds that are given in terms of various graph structural parameters like the number of vertices, the number of edges, the matching number, the chromatic number, the maximum degree, and the number of spanning trees [5-20], but also bounds for some special interesting classes of graphs, such as circulant graphs, unicyclic graphs, and bicyclic graphs [21-27]. Along this line, we consider the relation between the Kirchhoff index and the independence number as well as the clique number, and bounds are obtained for the Kirchhoff index of graphs via the two graph invariants. In addition, lower and upper bounds for the Kirchhoff index of planar graphs and fullerene graphs are investigated. For more information on the Kirchhoff index of graphs, the readers are referred to the most recent papers [28-36] and references therein.

\section{General Bounds}

2.1. A Lower Bound via the Independence Number. We first introduce some notations. Denote the vertex set and edge set of $G$, respectively, by $V(G)$ and $E(G)$. A subset $X$ of $V(G)$ is called independent if its vertices are mutually nonadjacent. The independence number $\alpha(G)$ is the largest cardinality among all independent sets of $G$. The clique number $\omega(G)$ of $G$ is the largest set of mutually adjacent vertices in $G$. The degree of vertex $i$, denoted by $d_{i}(G)$, is the number of neighbors of $i$. The adjacency matrix $A(G)$ of $G$ is an $n \times n$ matrix with the $(i, j)$-entry equal to 1 if vertices $i$ and $j$ are adjacent and 0 otherwise. Let $D(G)=\operatorname{diag}\left(d_{1}(G), d_{2}(G), \ldots, d_{n}(G)\right)$ be the diagonal matrix of vertex degrees. Then the Laplacian matrix of $G$ is $L(G)=D(G)-A(G)$. Let $\lambda_{0} \leq \lambda_{1} \leq \cdots \leq \lambda_{n-1}$ 
be the eigenvalues of $L(G)$, called the Laplacian eigenvalues of $G$. Since $G$ is connected, $\lambda_{0}=0$ and $\lambda_{k}>0$ for $k=1,2$, $\ldots, n-1$ [37]. The spectrum of $L(G)$, also known as the Laplacian spectrum of $G$, is

$$
S(G)=\left(\lambda_{0}, \lambda_{1}, \ldots, \lambda_{n-1}\right) .
$$

Zhu et al. [38] and Gutman and Mohar [39] established a classical result for computing the Kirchhoff index via the Laplacian spectrum of $G$.

\section{Theorem 1.}

$$
K f(G)=n \sum_{k=1}^{n-1} \frac{1}{\lambda_{k}} .
$$

The join of $G_{1}$ and $G_{2}$, denoted by $G_{1}+G_{2}$, is the graph obtained from $G_{1} \cup G_{2}$ by adding all edges between vertices of $G_{1}$ and that of $G_{2}$, where $\cup$ denote the disjoint union. The Laplacian eigenvalues of $G_{1}+G_{2}$ are characterized in the following result.

Lemma 2 (see [37]). Let $G_{1}$ and $G_{2}$ be graphs such that $\left|V\left(G_{1}\right)\right|=n$ and $\left|V\left(G_{2}\right)\right|=m$. Suppose that $0=\lambda_{0} \leq \lambda_{1} \leq$ $\lambda_{2} \leq \cdots \leq \lambda_{n-1}$ and $0=\mu_{0} \leq \mu_{1} \leq \mu_{2} \leq \cdots \leq \mu_{m-1}$ are Laplacian eigenvalues of $G_{1}$ and $G_{2}$. Then the Laplacian eigenvalues of $G_{1}+G_{2}$ are $0, m+n, m+\lambda_{i}, n+\mu_{j}, i=1,2, \ldots$, $n-1, j=1,2, \ldots, m-1$.

The nonincreasing property of the Kirchhoff index, as stated blow, plays an important role in estimating bounds for the Kirchhoff index.

Lemma 3 (see [12]). Let $G$ be a noncomplete graph. If $G^{\prime}$ is obtained from $G$ by adding an edge, then $K f\left(G^{\prime}\right)<K f(G)$.

For simplicity, if there is no confusion, we always abbreviate $r_{i j}(G), d_{i j}(G), d_{v}(G), \alpha(G)$, and $\omega(G)$ to $r_{i j}, d_{i j}, d_{v}, \alpha$, and $\omega$, respectively. Throughout the paper, we use $K_{n}, P_{n}$ to denote the complete and path graph of order $n$, respectively. We use $\bar{G}$ to denote the complement of $G$. Then the main result of this subsection is given as follows.

Theorem 4. Let $G$ be a connected graph with $n$ vertices and independence number $\alpha$. Then

$$
K f(G) \geq n-\alpha+\frac{n(\alpha-1)}{n-\alpha},
$$

with equality if and only if $G \cong \overline{K_{\alpha}}+K_{n-\alpha}$.

Proof. Let $G_{0}$ be a graph having the minimum Kirchhoff index among all connected graphs with $n$ vertices and independence number $\alpha$. Then by the nonincreasing property of the Kirchhoff index as given in Lemma 3, it is easily seen that $G_{0} \cong \overline{K_{\alpha}}+K_{n-\alpha}$. Now we compute the Kirchhoff index of $G_{0}$. Since it is well known that $S\left(\overline{K_{\alpha}}\right)=(0,0, \ldots, 0)$ and $S\left(K_{n-\alpha}\right)=(n-\alpha, n-\alpha, \ldots, n-\alpha)$, by Lemma 2 , we get

$$
S\left(G_{0}\right)=(0, \underbrace{n-\alpha, \ldots, n-\alpha}_{\alpha-1}, \underbrace{n, \ldots, n}_{n-\alpha}) \text {. }
$$

Then according to Theorem 1 , it follows that

$$
K f\left(G_{0}\right)=n\left(\frac{\alpha-1}{n-\alpha}+\frac{n-\alpha}{n}\right)=n-\alpha+\frac{n(\alpha-1)}{n-\alpha} .
$$

2.2. An Upper Bound via the Clique Number. Let $\mathscr{G}_{\omega}$ be the set of graphs with $n$ vertices and clique number $\omega$. Let $G_{p}$ denote the graph obtained by identifying one end vertex of path $P_{n-\omega+1}$ with any vertex of $K_{\omega}$. In the following, we have shown that, among all the graphs in $\mathscr{G}_{\omega}, G_{p}$ has the maximum Kirchhoff index. To this end, we need the following two lemmas.

Lemma 5 (see [40]). Let $G$ be a graph with a cut edge $e=x y$ and $G_{1}$ and $G_{2}$ the components of $G-e$ containing $x$ and $y$, respectively. Then

$$
\begin{aligned}
K f(G)= & K f\left(G_{1}\right)+K f\left(G_{2}\right)+\left|V\left(G_{2}\right)\right| K f_{x}\left(G_{1}\right) \\
& +\left|V\left(G_{1}\right)\right| K f_{y}\left(G_{2}\right)+\left|V\left(G_{1}\right)\right|\left|V\left(G_{2}\right)\right|,
\end{aligned}
$$

where $K f_{x}(G)$ denote the sum of resistance distances between $x$ and all the other vertices of $G$.

Lemma 6 (see [41]). Let $T$ be an $n$ vertex tree different from $P_{n}$ and $S_{n}$. Then

$$
W\left(S_{n}\right)<W(T)<W\left(P_{n}\right) .
$$

Theorem 7. Let $G \in \mathscr{G}_{\omega}$ be different from $G_{p}$. Then

$$
K f(G)<K f\left(G_{p}\right) .
$$

Proof. If $\omega=2$, then $G_{p}$ is a path. Noticing that the Kirchhoff index is equal to the Wiener index for trees, by Lemmas 3 and 6 , the assertion holds. In the following, we assume that $\omega \geq 3$.

Let $G_{0}$ be the graph with the maximum Kirchhoff index and let $H$ be the subgraph of $G_{0}$ such that $H \cong K_{\omega}$.

Claim 1 (every component of $G_{0}-H$ is connected to $H$ by only one edge). Suppose to the contrary that there exists a component of $G_{0}-H$ such that it is connected to $H$ with edges $e_{1}, e_{2}, \ldots, e_{k}(k>1)$. Let $G_{1}$ be the graph obtained from $G_{0}$ by the deletion of $e_{2}, e_{3}, \ldots, e_{k}$. Then it is easily seen that $G_{1} \in \mathscr{G}_{\omega}$ and $G_{1}$ is a proper spanning subgraph of $G_{0}$. Thus by Lemma 3, we have $K f\left(G_{1}\right)>K f\left(G_{0}\right)$, contradicting the property that $G_{0}$ has the maximum Kirchhoff index.

Claim 2 (every component of $G_{0}-H$ is a tree). Suppose to the contrary that there exists a component $C$ of $G_{0}-H$ such that $C$ is not a tree. Let $T$ be a spanning tree of $C$. Remove from $G_{0}$ all the edges in $C$ but not in $T$ to obtain $G_{1}$. Then $G_{1} \in \mathscr{G}_{\omega}$ and $G_{1}$ is a proper subgraph of $G_{0}$. Again by Lemma 3, we have $K f\left(G_{1}\right)>K f\left(G_{0}\right)$, a contradiction.

Claim 3 (every component of $G_{0}-H$ is a path, which connects to $H$ via an end vertex). Let $C$ be any component of $G_{0}-H$. By Claims 1 and 2, we know that $C$ is a tree and $C$ is connected to $H$ by a single edge $e=x y(y \in C)$. Clearly $G-e$ has two 
components, one is $C$, and denote the other one by $G_{1}$. Then by Lemma 5 ,

$$
\begin{aligned}
K f\left(G_{0}\right)= & K f\left(G_{1}\right)+K f(C)+|V(C)| K f_{x}\left(G_{1}\right) \\
& +\left|V\left(G_{1}\right)\right| K f_{y}(C)+\left|V\left(G_{1}\right)\right||V(C)| .
\end{aligned}
$$

Since $K f\left(G_{0}\right)$ has the maximum Kirchhoff index and by Lemma 6 and the fact that the Kirchhoff index is equal to the Wiener index for trees, we know that $K f\left(G_{0}\right)$ is maximized if and only if $C$ is a path and $y$ is an end vertex of $C$; the claim holds.

Claim 4 (every vertex of $H$ has degree at most $\omega$ in $G_{0}$. Suppose to the contrary that there exists $x \in H$ such that the degree of $x$ in $G_{0}$ is larger than $\omega$. Then by Claim $1, x$ is connected to at least two components of $G_{0}-H$. Let $C_{1}$ and $C_{2}$ be two such components connecting to $x$ by edges $x y_{1}$ and $x y_{2}$, respectively. By Claim 3 , both $C_{1}$ and $C_{2}$ are paths with $y_{1}$ and $y_{2}$ being end vertices of them. Without loss of generality, suppose that the length of $C_{1}$ is less than or equal to the length of $C_{2}$. Let $z_{1}$ (resp., $z_{2}$ ) be the end vertex of $C_{1}$ (resp., $C_{2}$ ) different from $y_{1}$ (resp., $y_{2}$ ), and let $u$ be the unique neighbor of $z_{1}$. Now construct a new graph $G_{1}$ from $G_{0}$ by first deleting the edge $z_{1} u$ and then adding a new edge $z_{1} z_{2}$. Clearly $G_{1} \in \mathscr{G}_{\omega}$. We show $K f\left(G_{0}\right)<K f\left(G_{1}\right)$ so that Claim 4 is proved by contradiction. It is easily seen that, for any two vertices $u, v$ distinct from $z_{1}$,

$$
r_{u v}\left(G_{0}\right)=r_{u v}\left(G_{1}\right) .
$$

Thus to show $K f\left(G_{0}\right)<K f\left(G_{1}\right)$, we need only to show that $K f_{z_{1}}\left(G_{0}\right)<K f_{z_{1}}\left(G_{1}\right)$. On one hand, it is easily verified that

$$
\sum_{a \in\left(V\left(C_{1}\right) \cup V\left(C_{2}\right) \cup\{x\}\right)} r_{z_{1} a}\left(G_{0}\right)=\sum_{a \in\left(V\left(C_{1}\right) \cup V\left(C_{2}\right) \cup\{x\}\right)} r_{z_{1} a}\left(G_{1}\right) .
$$

On the other hand, for any vertex $b$ that is not contained in $\left(V\left(C_{1}\right) \cup V\left(C_{2}\right) \cup\{x\}\right)$, we have

$$
\begin{aligned}
r_{z_{1} b}\left(G_{0}\right) & =r_{z_{1} x}\left(G_{0}\right)+r_{x b}\left(G_{0}\right)<r_{z_{1} x}\left(G_{1}\right)+r_{x b}\left(G_{0}\right) \\
& =r_{z_{1} x}\left(G_{1}\right)+r_{x b}\left(G_{1}\right)=r_{z_{1} b}\left(G_{1}\right) .
\end{aligned}
$$

Hence it follows that $K f_{z_{1}}\left(G_{0}\right)<K f_{z_{1}}\left(G_{1}\right)$, as required.

Claim 5 ( $G-H$ has only one component). Suppose to the contrary that $G-H$ has at least two components $C_{1}$ and $C_{2}$. Then by Claim $4, C_{1}$ and $C_{2}$ must be connected with $H$ via different vertices of $H$, say $x$ and $y$. Let $e_{1}=x u$ and $e_{2}=y v$ be the two edges connecting $H$ with $C_{1}$ and $C_{2}$, respectively. Then both $C_{1}$ and $C_{2}$ are paths with $u$ and $v$ being their end vertices. Suppose that the lengths of $C_{1}$ and $C_{2}$ are $l_{1}$ and $l_{2}$, respectively. Without loss of generality, we may assume that $l_{1} \leq l_{2}$. Let $z_{1}$ (resp., $z_{2}$ ) be the other end vertex of $C_{1}$ (resp., $C_{2}$ ) different from $u$ (resp., $v$ ), and let $z$ be the unique neighbor of $z_{1}$. Construct a new graph $G_{1}$ from $G_{0}$ by first deleting the edge $z z_{1}$ and then adding a new edge between $z_{1}$ and $z_{2}$. Now we show $K f\left(G_{0}\right)<K f\left(G_{1}\right)$, which thus gives the desired contradiction. For any two vertices $p, q$ distinct from $z_{1}$, it is easily seen that

$$
r_{p q}\left(G_{0}\right)=r_{p q}\left(G_{1}\right) .
$$

Thus it suffices to show that $K f_{z_{1}}\left(G_{0}\right)<K f_{z_{1}}\left(G_{1}\right)$. On one hand,

$$
\begin{aligned}
& \sum_{a \in V\left(C_{1}\right) \cup V\left(C_{2}\right) \cup\{x, y\}} r_{z_{1} a}\left(G_{0}\right) \\
= & 1+2+\cdots+l_{1}+\left(l_{1}+\frac{2}{\omega}\right) \\
& +\left(l_{1}+1+\frac{2}{\omega}\right)+\left(l_{1}+2+\frac{2}{\omega}\right)+\cdots+\left(l_{1}+1_{2}+\frac{2}{\omega}\right),
\end{aligned}
$$

while

$$
\begin{gathered}
\sum_{a \in V\left(C_{1}\right) \cup V\left(C_{2}\right) \cup\{x, y\}} r_{z_{1} a}\left(G_{1}\right) \\
=1+2+\cdots+l_{2}+\left(l_{2}+1\right)+\left(l_{2}+1+\frac{2}{\omega}\right) \\
+\left(l_{2}+2+\frac{2}{\omega}\right)+\cdots+\left(l_{2}+1_{1}+\frac{2}{\omega}\right),
\end{gathered}
$$

which implies that

$$
\sum_{a \in V\left(C_{1}\right) \cup V\left(C_{2}\right) \cup\{x, y\}} r_{z_{1} a}\left(G_{0}\right)<\sum_{a \in V\left(C_{1}\right) \cup V\left(C_{2}\right) \cup\{x, y\}} r_{z_{1} a}\left(G_{1}\right) .
$$

On the other hand, for any vertex $b$ that is not contained in $\left(V\left(C_{1}\right) \cup V\left(C_{2}\right) \cup\{x, y\}\right)$, we have

$$
\begin{aligned}
r_{z_{1} b}\left(G_{0}\right) & =r_{z_{1} x}\left(G_{0}\right)+r_{x b}\left(G_{0}\right)<r_{z_{1} y}\left(G_{1}\right)+r_{x b}\left(G_{0}\right) \\
& =r_{z_{1} x}\left(G_{1}\right)+r_{y b}\left(G_{1}\right)=r_{z_{1} b}\left(G_{1}\right) .
\end{aligned}
$$

Hence it follows that $K f_{z_{1}}\left(G_{0}\right)<K f_{z_{1}}\left(G_{1}\right)$ as required.

From Claims 1-5, we deduce that $G_{0} \cong G_{p}$, as desired.

By Lemma 5, simple calculation leads to

$$
\begin{aligned}
K f\left(G_{p}\right) & \\
= & \frac{(n-\omega)^{3}-(n-\omega)}{6}+\frac{\omega(n-\omega)(n-\omega-1)}{2} \\
& +\frac{2(\omega-1)(n-\omega)}{\omega}+\omega-1+\omega(n-\omega) \\
= & (n-\omega)\left(\frac{n^{2}+n \omega-2 \omega^{2}+3 \omega+11}{6}-\frac{2}{\omega}\right)+\omega-1 .
\end{aligned}
$$

Consequently, the upper bound on the Kirchhoff index is given in terms of the clique number. 
Theorem 8. Let $G$ be a connected graph with $n$ vertices and clique number $\omega$. Then

$$
K f(G) \leq(n-\omega)\left(\frac{n^{2}+n \omega-2 \omega^{2}+3 \omega+11}{6}-\frac{2}{\omega}\right)+\omega-1
$$

with equality holds if and only if $G \cong G_{p}$.

\section{Planar Graphs}

A planar graph is a graph which can be drawn in the plane without edges crossing. In this section, we investigate bounds for Kirchhoff index of planar graphs. The following lemma is used.

Lemma 9 (see [17]). $\lambda_{1}=\lambda_{2}=\cdots \lambda_{n-1}$ if and only if $G \cong K_{n}$.

Theorem 10. For an $n$ vertex planar graph $G(n \geq 3)$,

$$
\frac{n(n-1)^{2}}{6(n-2)} \leq K f(G) \leq \frac{n^{3}-n}{6} \text {. }
$$

The first equality holds if and only if $G \cong K_{3}, K_{4}$, and the second does if and only if $G$ is a path.

Proof. The upper bound is well known and we suffice to show the lower bound. Bearing in mind that, for a planar graph $G$, $|E(G)| \leq 3 n-6$, together with the fact that $\sum_{i=1}^{n} \lambda_{i}=\sum_{i=1}^{n-1} \lambda_{i}=$ $\sum_{i=1}^{n} d_{i}=2|E(G)|$, by Cauchy-Schwarz inequality, we have

$$
\sum_{i=1}^{n-1} \frac{1}{\lambda_{i}} \geq \frac{(n-1)^{2}}{2|E(G)|} \geq \frac{(n-1)^{2}}{2(3 n-6)}=\frac{(n-1)^{2}}{6(n-2)}
$$

The equality holds if and only if $1 / \lambda_{1}=1 / \lambda_{2}=\cdots 1 / \lambda_{n-1}$, that is, $\lambda_{1}=\lambda_{2}=\cdots=\lambda_{n-1}$, which implies by Lemma 9 that $G$ is a complete graph. Then the proof is completed by noticing that only $K_{3}$ and $K_{4}$ are planar complete graphs for $n \geq 3$.

Though the lower bound is not sharp for $n \geq 5$, it can be shown that, up to a scale factor, the bound is asymptotically attainable. One example is the star graph $S_{n}$, which has Kirchhoff index $(n-1)^{2}$. This indicates that the lower bound can be asymptotically attained up to a scale factor of at least $1 / 6$. In fact, the scale factor could be improved to at least $\sqrt{3} / 6$. For example, consider the planar graph $G=K_{2}+P_{n-2}$. Since the Laplacian eigenvalues of $P_{n}$ are [42]

$$
4 \sin ^{2} \frac{k \pi}{2 n}, \quad k=0,1, \ldots, n-1,
$$

by Lemma 2, we readily obtain that the Laplacian eigenvalues of $G$ are

$$
0, n, n, 2+4 \sin ^{2} \frac{k \pi}{2(n-2)}, \quad k=1,2, \ldots, n-3
$$

Therefore, by Theorem 1 , we have

$$
\begin{aligned}
K f(G) & =2+\frac{n}{2} \sum_{k=1}^{n-3} \frac{1}{1+2 \sin ^{2}(k \pi / 2(n-2))} \\
& =2+\frac{n}{2} \sum_{k=1}^{n-3} \frac{1}{2-\cos (k \pi /(n-2))} .
\end{aligned}
$$

Now we consider the asymptotic behavior of $K f(G)$ as $n \rightarrow$ $\infty$ :

$$
\begin{aligned}
\lim _{n \rightarrow \infty} & \frac{1}{n^{2}} K f\left(T_{n}\right) \\
& =\lim _{n \rightarrow \infty}\left(2+\frac{n}{2} \sum_{k=1}^{n-3} \frac{1}{2-\cos (k \pi /(n-2))}\right) \\
& =\lim _{n \rightarrow \infty} \frac{2}{n^{2}}+\lim _{n \rightarrow \infty} \frac{1}{2 n} \sum_{k=1}^{n-3} \frac{1}{2-\cos (k \pi /(n-2))} \\
& =\lim _{n \rightarrow \infty} \frac{n-3}{2 n} \sum_{k=1}^{n-3} \frac{1}{2-\cos (k \pi /(n-2))} \frac{1}{n-3} \\
& =\frac{1}{2} \lim _{n \rightarrow \infty} \sum_{k=1}^{n-3} \frac{1}{2-\cos (k \pi /(n-2))} \frac{1}{n-3} \\
= & \frac{1}{2} \int_{0}^{1} \frac{1}{2-\cos x \pi} \mathrm{d} x \\
= & \frac{1}{2} \frac{\sqrt{3}}{3}=\frac{\sqrt{3}}{6} .
\end{aligned}
$$

Hence $K f\left(T_{n}\right)$ grows as $(\sqrt{3} / 6) n^{2}$ as $n \rightarrow \infty$, and the scale factor is improved to at least $\sqrt{3} / 6$.

\section{Fullerene Graphs}

A fullerene graph $G$ is a cubic 3-connected planar graph with exactly 12 pentagons and other hexagons. Fullerene graphs are well studied in both mathematical and chemical literatures. To give bounds for fullerene graphs, we need some preparations.

The famous Foster first formula, given by Foster [43], states that

$$
\sum_{i \sim j} r_{i j}=n-1
$$

where $i \sim j$ means $i$ and $j$ are adjacent. Foster's second formula [44], also given by Foster, perhaps less well known, states that

$$
\sum_{v} \frac{r_{i j}}{d_{v}}=n-2,
$$

where $r_{i j}$ is measured across the end vertices of two adjacent edges $i v$ and $v j$ and the sum is taken over all adjacent edges. Palacios [14] extended Foster's first and second formulae and obtained the so-called Foster third formula, which states that

$$
\sum_{v, w} \sum_{i<j} \frac{r_{i j}}{d_{v} d_{w}}=n-3+\sum_{j} \frac{1}{d_{j}} \sum_{k \sim j} \frac{1}{d_{k}},
$$


where the sum is taken over all pairs of vertices $i$ and $j$ such that $i v w j$ is a 3 -walk. In particular, if $G$ is $k$ regular, then the above equation can be simply written as

$$
\sum_{3 \text {-walks }} r_{i j} \kappa_{i j}=(n-3) k^{2}+n k \text {, }
$$

where $\kappa_{i j}$ is the number of 3 -walks from $i$ to $j$.

Now we give lower bounds for resistance distance between any two nonadjacent vertices in $G$ in terms of the distance between them.

Lemma 11. Let $G$ be a fullerene graph and let $i, j \in V(G)$. Suppose that $d_{i j}=d \geq 2$. Then

$$
r_{i j} \geq \begin{cases}\frac{2}{3}\left(2-2^{1-(d / 2)}\right), & \text { if } d \text { is even } \\ \frac{2}{3}\left(2-2^{(3-d) / 2}+2^{(-1-d) / 2}\right), & \text { otherwise. }\end{cases}
$$

Proof. If $d=2$, by the inequality $r_{i j} \geq 1 / d_{i}+1 / d_{j}$ [45], we know that $r_{i j} \geq 1 / 3+1 / 3=2 / 3$, as desired. Now suppose that $d \geq 3$. We distinguish the following two cases.

Case 1 ( $d$ is even). We partition vertices in $V(G) \backslash\{i, j\}$ into the following parts. For $k=1,2, \ldots,(d / 2)-1$, let

$$
\begin{aligned}
& I_{k}=\left\{x \mid x \in V(G) \backslash\{i, j\}, d_{i x}=k\right\}, \\
& J_{k}=\left\{x \mid x \in V(G) \backslash\{i, j\}, d_{j x}=k\right\},
\end{aligned}
$$

and let $I_{d / 2}$ be the set of the remaining vertices. For $k=1,2$, $\ldots, d / 2$, contract $I_{k}$ into a single vertex $i_{k}$. For $k=1,2, \ldots$, $(d / 2)-1$, contract $J_{k}$ into a single vertex $j_{k}$. Then we obtain a path $P$ of length $d$ with multiple edges;

$$
P:(i=) i_{0}, i_{1}, \ldots, i_{(d / 2)-1}, i_{d / 2}, j_{(d / 2)-1}, \ldots, j_{1}, j_{0}(=j) .
$$

According to the structure of $G$, it is easily seen that, for $k=1,2, \ldots,(d / 2)-1,\left|I_{k}\right| \leq 3 \times 2^{k-1},\left|J_{k}\right| \leq 3 \times 2^{k-1}$. For $k=1,2, \ldots,(d / 2)-1$, since every vertex in $I_{k}$ has at least on neighbor in $I_{k-1}$, the number of edges connecting $i_{k}$ and $i_{k+1}$ is no more than $2\left|I_{k}\right| \leq 3 \times 2^{k}$. Similarly, for $k=1,2, \ldots$, $(d / 2)-2$, the number of edges connecting $j_{k}$ and $j_{k+1}$ is no more than $3 \times 2^{k}$, and the number of edges connecting $j_{(d / 2)-1}$ and $i_{d / 2}$ is no more than $3 \times 2^{d-1}$. Hence, according to the series and parallel connection rules of resistors, we know that

$$
\begin{aligned}
r_{i j}(P)= & r_{i_{0}, i_{1}}(P)+r_{i_{1}, i_{2}}(P)+\cdots+r_{i_{(d / 2)-1}, i_{d / 2}}(P) \\
& +r_{j_{(d / 2)-1}, i_{d / 2}}(P)+r_{j_{(d / 2)-2}, j_{(d / 2)-1}}(P) \\
& +\cdots+r_{j_{1}, j_{2}}(P)+r_{j_{0}, j_{1}}(P) \\
\geq & \frac{1}{3}+\frac{1}{3 \times 2}+\cdots+\frac{1}{3 \times 2^{(d / 2)-1}}+\frac{1}{3 \times 2^{(d / 2)-1}} \\
& +\frac{1}{3 \times 2^{(d / 2)-2}}+\cdots+\frac{1}{3 \times 2}+\frac{1}{3} \\
= & \frac{2}{3}\left(2-2^{1-(d / 2)}\right) .
\end{aligned}
$$

Since there are exactly $6 n-60$ pairs of vertices at distance 3 and by Lemma 11 we know that $r_{i j} \geq 5 / 6$ for $d_{i j}=3$, hence

$$
\sum_{\substack{i<j \\ d_{i j}=3}} r_{i j} \geq \frac{5}{6}\left(\frac{9 n}{2}-30\right)=\frac{15 n}{4}-25 .
$$

Then by Rayleigh's short-cut principle [46], which states that shorting certain vertices together can only decrease the resistance distances between two given vertices, whereas cutting certain edges can only increase the resistance distance between two given vertices, it follows that

$$
r_{i j}(G) \geq r_{i j}(P) \geq \frac{2}{3}\left(2-2^{1-(d / 2)}\right) .
$$

Case 2 ( $d$ is odd). First partition vertices in $V(G) \backslash\{i, j\}$ in the following way. For $k=1,2, \ldots,(d-1) / 2-1$, let

$$
I_{k}=\left\{x \mid x \in V(G) \backslash\{i, j\}, d_{i x}=k\right\},
$$

for $k=1,2, \ldots,(d-1) / 2$, let

$$
J_{k}=\left\{x \mid x \in V(G) \backslash\{i, j\}, d_{j x}=k\right\},
$$

and let $I_{(d-1) / 2}$ be the set of remaining vertices. Contract $I_{k}$ to a single vertex $i_{k}$ and contract $J_{k}$ to a single vertex $j_{k}, k=$ $1,2, \ldots,(d-1) / 2$. Using the same argument as the proof of Case 1 , we could obtain that $r_{i j} \geq(2 / 3)\left(2-2^{(3-d) / 2}+2^{(-1-d) / 2}\right)$.

The following result is useful.

Lemma 12 (see [47]). There are exactly $(9 n / 2-30)$ pairs of vertices at distance 3 in $G$.

Next we introduce a classical result in graph theoryMenger's Theorem.

Theorem 13 (see [48], Menger's Theorem). Let $G$ be an undirected graph, and let $u$ and $v$ be nonadjacent vertices in $G$. Then, the maximum number of pairwise internally disjoint $(u, v)$ paths in $G$ equals the minimum number of vertices from $V(G)-\{u, v\}$ whose deletion separates $u$ and $v$.

Now we are ready for our main result.

Theorem 14. For an $n$ vertex fullerene graph $G$, one has

$$
\frac{n^{2}}{2}-\frac{7 n}{4}-2 \leq K f(G)<\frac{n^{3}}{18}-n^{2}+\frac{329 n}{18}-\frac{92}{3} .
$$

Proof. We first prove the lower bound. By Foster's first formula,

$$
\sum_{\substack{i<j \\ d_{i j}=1}} r_{i j}=n-1 .
$$

Since $G$ is triangle free, it is obvious that the end vertices of any two adjacent edges in $G$ are at distance 2 . Hence by Foster's second formula,

$$
\sum_{\substack{i<j \\ d_{i j}=2}} r_{i j}=3(n-2) .
$$
列 
Since for $d_{i j} \geq 4$, by Lemma $11, r_{i j} \geq 1$, we have

$$
\begin{gathered}
\sum_{\substack{i<j \\
d_{i j} \geq 4}} r_{i j} \geq\left[\left(\begin{array}{l}
n \\
2
\end{array}\right)-\frac{3 n}{2}-3 n-\left(\frac{9 n}{2}-30\right)\right] \\
\times 1=\frac{n^{2}-19 n}{2}+30 .
\end{gathered}
$$

Hence

$$
\begin{aligned}
K f(G)= & \sum_{i<j} r_{i j}=\sum_{\substack{i<j \\
d_{i j}=1}} r_{i j}+\sum_{\substack{i<j \\
d_{i j}=2}} r_{i j} \\
& +\sum_{\substack{i<j \\
d_{i j}=3}} r_{i j}+\sum_{\substack{i<j \\
d_{i j} \geq 4}} r_{i j} \\
\geq & n-1+3 n-2+\frac{15 n}{4}-25+\frac{n^{2}-19 n}{2}+30 \\
= & \frac{n^{2}}{2}-\frac{7 n}{4}-2 .
\end{aligned}
$$

For the upper bound, we consider any two nonadjacent vertices $i$ and $j$. Since $G$ is 3-connected, by Menger's Theorem, $i$ and $j$ are connected by at least three pairwise internally disjoint paths. Suppose that $P_{1}, P_{2}$, and $P_{3}$ are three pairwise internally disjoint paths connecting $i$ and $j$. We consider the graph $G^{*}$ induced by $P_{1}, P_{2}$, and $P_{3}$. By Rayleigh's short-cut principle, $r_{i j}(G) \leq r_{i j}\left(G^{*}\right)$. Suppose that the lengths of $P_{1}, P_{2}$, and $P_{3}$ are $l_{1}, l_{2}$, and $l_{3}$, respectively. Then by the series and parallel connection rules of resistors,

$$
r_{i j}\left(G^{*}\right)=\frac{1}{\left(1 / l_{1}\right)+\left(1 / l_{2}\right)+\left(1 / l_{3}\right)}
$$

Since it is obvious that $l_{1}+l_{2}+l_{3} \leq n+1$, it follows that

$$
\frac{1}{l_{1}}+\frac{1}{l_{2}}+\frac{1}{l_{3}} \geq \frac{3}{n+1}+\frac{3}{n+1}+\frac{3}{n+1}=\frac{9}{n+1} .
$$

Thus $r_{i j}(G) \leq r_{i j}\left(G^{*}\right) \leq(n+1) / 9$.

By Foster's third formula (30) and noticing that there exist 3-walks which are not 3-path in $G$, we conclude that

$$
\sum_{\substack{i<j \\ d_{i j}=3}} r_{i j}<\sum_{3 \text {-walks }} r_{i j} \kappa_{i j}=(n-3) 3^{2}+3 n=12 n-27 .
$$

Hence

$$
\begin{aligned}
K f(G) & =\sum_{i<j} r_{i j} \\
& =\sum_{\substack{i<j \\
d_{i j}=1}} r_{i j}+\sum_{\substack{i<j \\
d_{i j}=2}} r_{i j}+\sum_{\substack{i<j \\
d_{i j}=3}} r_{i j}+\sum_{\substack{i<j \\
d_{i j} \geq 4}} r_{i j}
\end{aligned}
$$

$$
\begin{aligned}
< & n-1+3(n-2)+12 n-27 \\
& +\left(\frac{n^{2}-19 n}{2}+30\right) \times \frac{n+1}{9} \\
= & \frac{n^{3}}{18}-n^{2}+\frac{329 n}{18}-\frac{92}{3} .
\end{aligned}
$$

\section{Conflict of Interests}

The author declares that there is no conflict of interests regarding the publication of this article.

\section{Acknowledgments}

The author would like to thank the anonymous referee for his/her careful reading of the paper and valuable comments and suggestions. The support of National Natural Science Foundation of China through Grants no. 11201404 and 11371307, China Postdoctoral Science Foundation through Grants no. 2012M521318 and 2013T60662, and Special Funds for Postdoctoral Innovative Projects of Shandong Province through Grant no. 201203056 is greatly acknowledged.

\section{References}

[1] D. J. Klein and M. Randić, "Resistance distance," Journal of Mathematical Chemistry, vol. 12, no. 1-4, pp. 81-95, 1993.

[2] B. Zhou and N. Trinajstić, "Mathematical properties of molecular descriptors based on distances," Croatica Chemica Acta, vol. 83, pp. 227-242, 2010.

[3] H. Wiener, "Structural determination of paraffin boiling points," Journal of the American Chemical Society, vol. 69, no. 1, pp. 1720, 1947.

[4] D. Babić, D. J. Klein, I. Lukovits, S. Nikolić, and N. Trinajstić, "Resistance-distance matrix: a computational algorithm and its application," International Journal of Quantum Chemistry, vol. 90, no. 1, pp. 166-176, 2002.

[5] M. Bianchi, A. Cornaro, J. L. Palacios, and A. Torriero, "Bounds for the Kirchhoff index via majorization techniques," Journal of Mathematical Chemistry, vol. 51, no. 2, pp. 569-587, 2013.

[6] A. Cornaro and G. P. Clemente, "A new lower bound for the Kirchhoff index using a numerical procedure based on majorization techniques," Electronic Notes in Discrete Mathematics, vol. 41, pp. 383-390, 2013.

[7] K. C. Das, "On the Kirchhoff index of graphs," Zeitschrift für Naturforschung, vol. 68, pp. 531-538, 2013.

[8] K. C. Das, A. D. Güngörr, and A. S. Çevik, "On Kirchhoff index and resistance-distance energy of a graph," MATCH: Communications in Mathematical and in Computer Chemistry, vol. 67, no. 2, pp. 541-556, 2012.

[9] K. C. Das, K. Xu, and I. Gutman, "Comparison between Kirchhoff index and the Laplacian-energy-like invariant," Linear Algebra and its Applications, vol. 436, no. 9, pp. 3661-3671, 2012.

[10] I. Gutman, D. Vidović, and B. Furtula, "Chemical applications of the Laplacian spectrum. VII. Studies of the Wiener and 
Kirchhoff indices," Indian Journal of Chemistry A: Inorganic, Physical, Theoretical and Analytical Chemistry, vol. 42, no. 6, pp. 1272-1278, 2003.

[11] R. Li, "Lower bounds for the Kirchhoff index," MATCH: Communications in Mathematical and in Computer Chemistry, vol. 70, no. 1, pp. 163-174, 2013.

[12] I. Lukovits, S. Nikolić, and N. Trinajstić, "Resistance distance in regular graphs," International Journal of Quantum Chemistry, vol. 71, no. 3, pp. 217-225, 1999.

[13] J. L. Palacios, "Closed-form formulas for Kirchhoff index," International Journal of Quantum Chemistry, vol. 81, pp. 135140, 2001.

[14] J. L. Palacios, "Foster's formulas via probability and the Kirchhoff index," Methodology and Computing in Applied Probability, vol. 6, no. 4, pp. 381-387, 2004.

[15] J. L. Palacios and J. M. Renom, "Bounds for the Kirchhoff index of regular graphs via the spectra of their random walks," International Journal of Quantum Chemistry, vol. 110, no. 9, pp. 1637-1641, 2010.

[16] J. L. Palacios and J. M. Renom, "Broder and Karlin's formula for hitting times and the Kirchhoff Index," International Journal of Quantum Chemistry, vol. 111, no. 1, pp. 35-39, 2011.

[17] B. Zhou, "On sum of powers of the Laplacian eigenvalues of graphs," Linear Algebra and its Applications, vol. 429, no. 8-9, pp. 2239-2246, 2008.

[18] B. Zhou and N. Trinajstić, "A note on Kirchhoff index," Chemical Physics Letters, vol. 445, pp. 120-123, 2008.

[19] B. Zhou and N. Trinajstić, "The kirchhoff index and the matching number," International Journal of Quantum Chemistry, vol. 109, no. 13, pp. 2978-2981, 2009.

[20] B. Zhou and N. Trinajstić, "On resistance-distance and Kirchhoff index," Journal of Mathematical Chemistry, vol. 46, no. 1, pp. 283-289, 2009.

[21] L. Feng, G. Yu, K. Xu, and Z. Jiang, "A note on the Kirchhoff index of bicyclic graphs," Ars Combinatoria, vol. 114, pp. 33-40, 2014.

[22] Q. Guo, H. Deng, and D. Chen, "The extremal Kirchhoff index of a class of unicyclic graphs," MATCH Communications in Mathematical and in Computer Chemistry, vol. 61, pp. 713-722, 2009.

[23] H. Wang, H. Hua, and D. Wang, "Cacti with minimum, secondminimum, and third-minimum Kirchhoff indices," Mathematical Communications, vol. 15, no. 2, pp. 347-358, 2010.

[24] Y. Yang, "Bounds for the Kirchhoff index of bipartite graphs," Journal of Applied Mathematics, vol. 2012, Article ID 195242, 9 pages, 2012.

[25] W. Zhang and H. Deng, "The second maximal and minimal Kirchhoff indices of unicyclic graphs," MATCH: Communications in Mathematical and in Computer Chemistry, vol. 61, no. 3, pp. 683-695, 2009.

[26] H. Zhang, X. Jiang, and Y. Yang, "Bicyclic graphs with extremal Kirchhoff index," MATCH: Communications in Mathematical and in Computer Chemistry, vol. 61, no. 3, pp. 697-712, 2009.

[27] H. Zhang and Y. Yang, "Resistance distance and kirchhoff index in circulant graphs," International Journal of Quantum Chemistry, vol. 107, no. 2, pp. 330-339, 2007.

[28] Q. Deng and H. Chen, "On the Kirchhoff index of the complement of a bipartite graph," Linear Algebra and Its Applications, vol. 439, no. 1, pp. 167-173, 2013.

[29] Q. Deng and H. Chen, "On extremal bipartite unicyclic graphs," Linear Algebra and Its Applications, vol. 444, pp. 89-99, 2014.
[30] X. Gao, Y. Luo, and W. Liu, "Kirchhoff index in line, subdivision and total graphs of a regular graph," Discrete Applied Mathematics, vol. 160, no. 4-5, pp. 560-565, 2012.

[31] J. Liu, J. Cao, X. Pan, and A. Elaiw, "The Kirchhoff index of hypercubes and related complex networks," Discrete Dynamics in Nature and Society, vol. 2013, Article ID 543189, 7 pages, 2013.

[32] J. Liu, X. Pan, Y. Wang, and J. Cao, "The Kirchhoff index of folded hypercubes and some variant networks," Mathematical Problems in Engineering, vol. 2014, Article ID 380874, 9 pages, 2014.

[33] X. Kuang and W. Yan, "The Kirchhoff indices of some graphs," Journal of Jimei University, vol. 17, pp. 65-70, 2012.

[34] M. H. Shirdareh-Haghighi, Z. Sepasdar, and A. Nikseresht, "On the Kirchhoff index of graphs and some graph operations," Proceedings of the Indian Academy of Science. In press.

[35] W. Wang, D. Yang, and Y. Luo, "The Laplacian polynomial and Kirchhoff index of graphs derived from regular graphs," Discrete Applied Mathematics, vol. 161, no. 18, pp. 3063-3071, 2013.

[36] Z. You, L. You, and W. Hong, "Comment on 'Kirchhoff index in line, subdivision and total graphs of a regular graph," Discrete Applied Mathematics, vol. 161, no. 18, pp. 3100-3103, 2013.

[37] R. Merris, "Laplacian matrices of graphs: a survey," Linear Algebra and Its Applications, vol. 197-198, pp. 143-176, 1994.

[38] H. Y. Zhu, D. J. Klein, and I. Lukovits, "Extensions of the Wiener number," Journal of Chemical Information and Computer Sciences, vol. 36, no. 3, pp. 420-428, 1996.

[39] I. Gutman and B. Mohar, "The quasi-Wiener and the Kirchhoff indices coincide," Journal of Chemical Information and Computer Sciences, vol. 36, no. 5, pp. 982-985, 1996.

[40] O. Ivanciuc and D. J. Klein, "Building-block computation of Wiener-type indices for the virtual screening of combinatorial libraries," Croatica Chemica Acta, vol. 75, no. 2, pp. 577-601, 2002.

[41] R. C. Entringer, D. E. Jackson, and D. A. Snyder, "Distance in graphs," Czechoslovak Mathematical Journal, vol. 26, no. 2, pp. 283-296, 1976.

[42] W. N. Anderson and T. D. Morley, "Eigenvalues of the Laplacian of a graph," Linear and Multilinear Algebra, vol. 18, no. 2, pp. 141145, 1985.

[43] R. M. Foster, "The average impedance of an electrical network," in Contributions to Applied Mechanics, J. W. Edwards, Ed., pp. 333-340, Edwards Brothers, Ann Arbor, Mich, USA, 1949.

[44] R. M. Foster, "An extension of a network theorem," IRE Transactions on Circuit Theory, vol. 8, pp. 75-76, 1961.

[45] D. Coppersmith, P. Tetali, and P. Winkler, "Collisions among random walks on a graph," SIAM Journal on Discrete Mathematics, vol. 6, no. 3, pp. 363-374, 1993.

[46] P. G. Doyle and J. L. Snell, Random Walks and Electric Networks, vol. 22 of Carus Mathematical Monographs, Mathematical Association of America, Washington, DC, 1984.

[47] A. Behmaram, H. Yousefi-Azari, and A. R. Ashrafi, "Wiener polarity index of fullerenes and hexagonal systems," Applied Mathematics Letters, vol. 25, no. 10, pp. 1510-1513, 2012.

[48] K. Menger, "Zur allgemeinen Kurventhoerie," Fundamenta Mathematicae, vol. 10, pp. 96-115, 1927. 


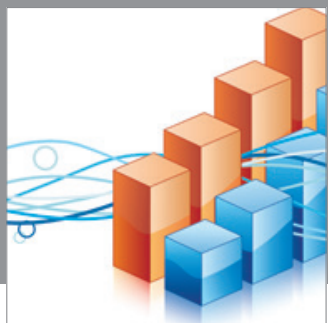

Advances in

Operations Research

mansans

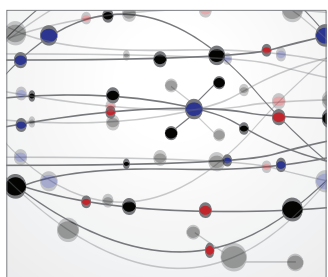

The Scientific World Journal
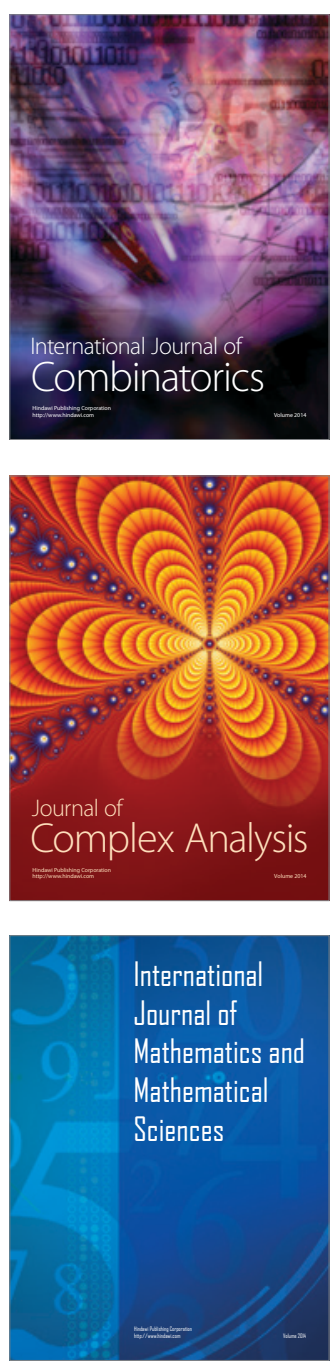
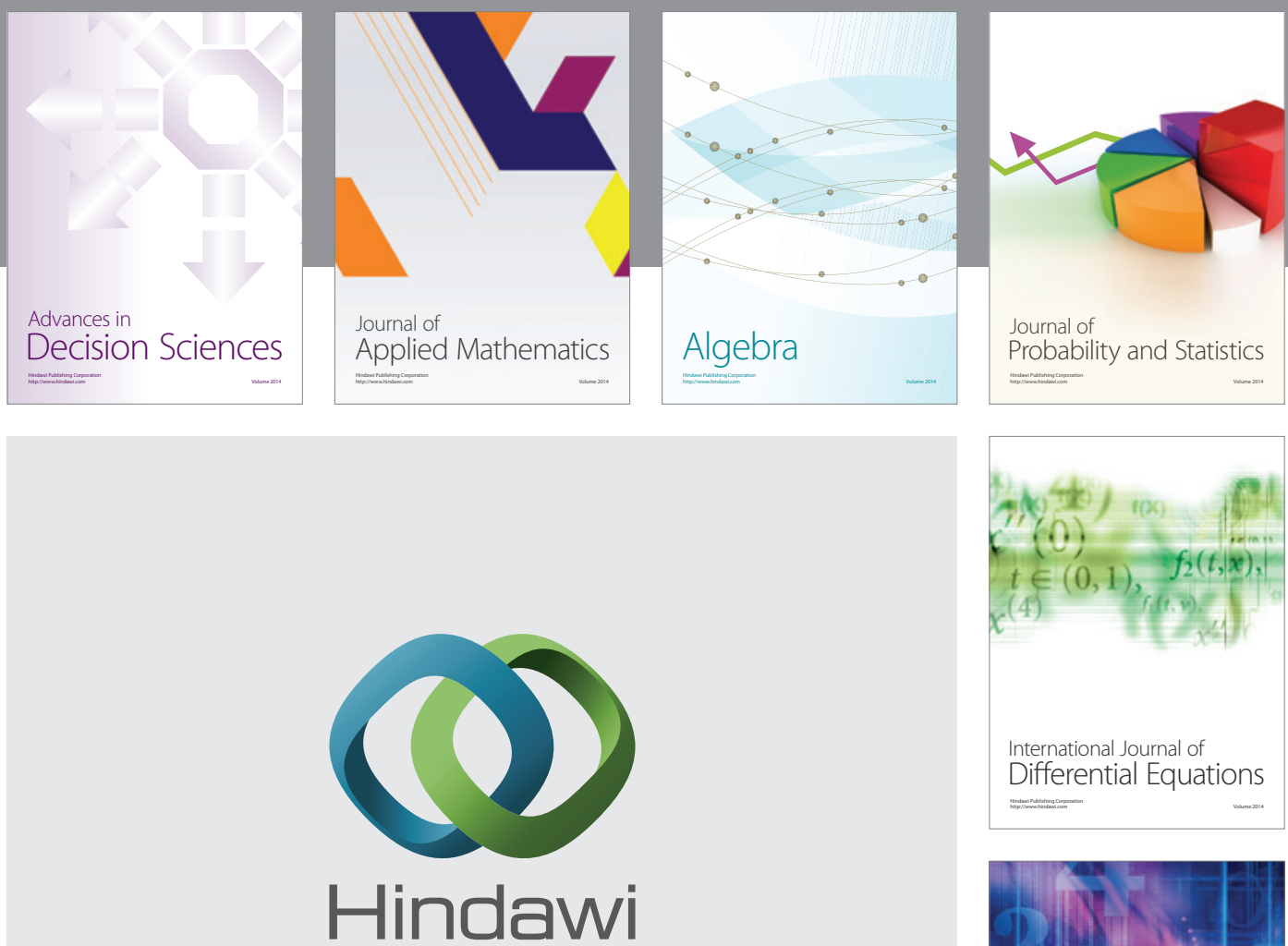

Submit your manuscripts at http://www.hindawi.com
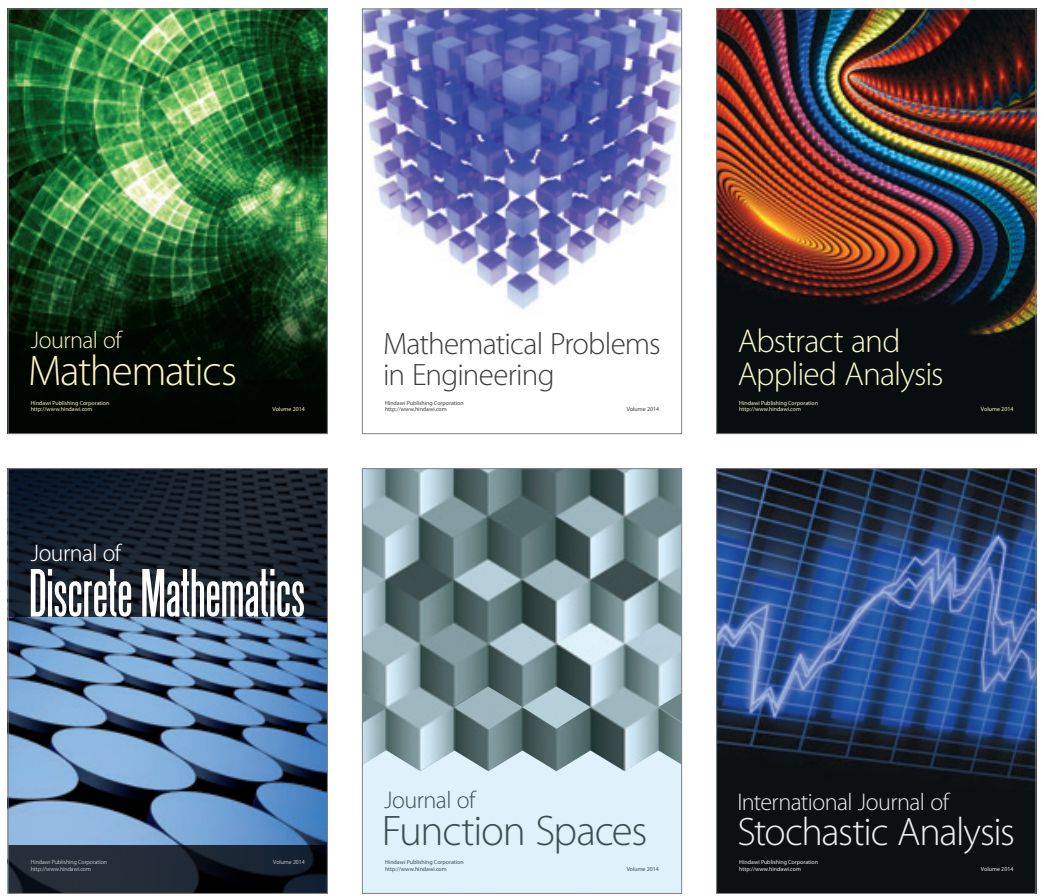

Journal of

Function Spaces

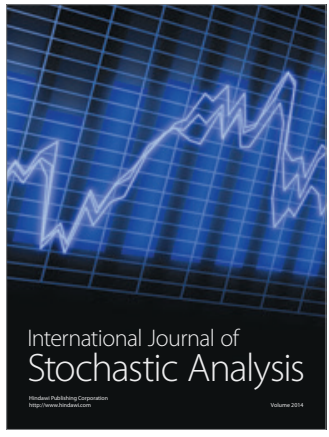

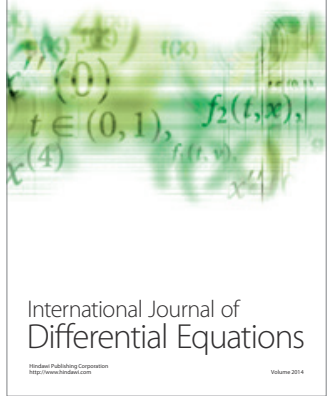
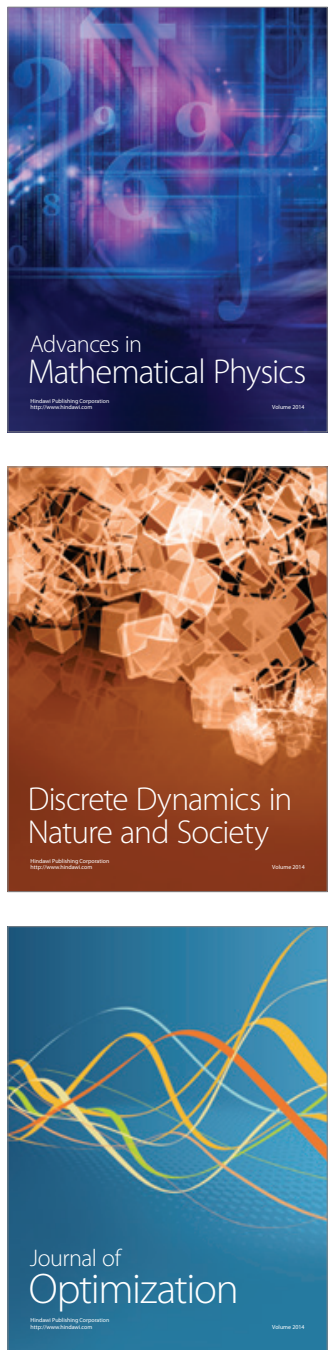